\title{
Impacts of oil spills on Arctic marine ecosystems: A quantitative and probabilistic risk assessment perspective
}

\author{
Helle, Inari
}

2020-02-18

Helle , I , Mäkinen , J A-E , Nevalainen , M K , Afenyo , M \& Vanhatalo , J 2020 , ' Impacts of oil spills on Arctic marine ecosystems: A quantitative and probabilistic risk assessment perspective ' , Environmental Science \& Technology , vol. 54 , no. 4 , pp. 2112-2121 . https://doi.org/10.1021/acs.es

http://hdl.handle.net/10138/313529

https://doi.org/10.1021/acs.est.9b07086

cc_by

publishedVersion

Downloaded from Helda, University of Helsinki institutional repository.

This is an electronic reprint of the original article.

This reprint may differ from the original in pagination and typographic detail.

Please cite the original version. 


\title{
Impacts of Oil Spills on Arctic Marine Ecosystems: A Quantitative and Probabilistic Risk Assessment Perspective
}

\author{
Inari Helle,* Jussi Mäkinen, Maisa Nevalainen, Mawuli Afenyo, and Jarno Vanhatalo*
}

Cite This: Environ. Sci. Technol. 2020, 54, 2112-2121

Read Online

ABSTRACT: Oil spills resulting from maritime accidents pose a poorly understood risk to the Arctic environment. We propose a novel probabilistic method to quantitatively assess these risks. Our method accounts for spatiotemporally varying population distributions, the spreading of oil, and seasonally varying species-specific exposure potential and sensitivity to oil. It quantifies risk with explicit uncertainty estimates, enables one to compare risks over large geographic areas, and produces information on a meaningful scale for decision-making. We demonstrate the method by assessing the short-term risks oil spills pose to polar bears, ringed seals, and walrus in the Kara Sea, the western part of the Northern Sea Route. The risks differ considerably between species, spatial locations, and seasons. Our
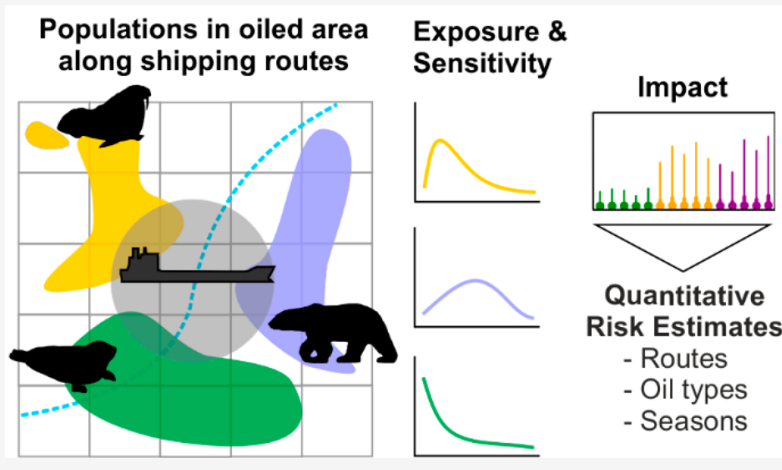
results support current aspirations to ban heavy fuel oil in the Arctic but show that we should not underestimate the risks of lighter oils either, as these oils can pollute larger areas than heavier ones. Our results also highlight the importance of spatially explicit season-specific oil spill risk assessment in the Arctic and that environmental variability and the lack of data are a major source of uncertainty related to the oil spill impacts.

\section{INTRODUCTION}

As climate change extends the ice-free period in high-latitude areas, the Arctic faces increasing maritime traffic and petroleum extraction activity. ${ }^{1}$ A growing number of oil drilling and shipping operations in areas experiencing harsh weather and extensive sea ice raises concerns about oil-related accidents. An accident similar to, for example, the Exxon Valdez oil spill in Alaska (1989) could have disastrous impacts on relatively pristine Arctic ecosystems. To proactively manage the risks maritime accidents pose to Arctic marine ecosystems, we need methods to assess them in a quantitative and spatiotemporally explicit manner while at the same time acknowledging high uncertainties typical for the Arctic. Despite this self-evident need, the risks have previously been described mainly in qualitative terms, and the handling of uncertainty has been limited. ${ }^{2}$ Recently, however, new quantitative approaches addressing the risks have started to emerge, ${ }^{3-5}$ and our work continues the general development of new methods suitable to Arctic risk assessment.

Oil spill risk assessment (OSRA) combines the probability of spill occurrence with an analysis of the consequences. The latter covers information about the oil's properties, its fate and movement, and its impacts on natural resources. ${ }^{6,7}$ However, many of these factors are difficult to assess reliably in the Arctic. Although there are sophisticated oil spill models to assess the trajectory and fate of spilled oil ${ }^{8}$ (e.g., SIMAP $^{9}$ and $\operatorname{OSCAR}^{10}$ ), they usually require extensive data about bathymetry, weather conditions, and coastal habitat types, which are currently not available for the majority of Arctic marine areas. Typically, these models also have a limited capacity to handle the uncertainty in their projections, and only few can contend with the interaction between oil and ice, ${ }^{11}$ even though significant progress has been made in this area in recent years. ${ }^{12,13}$ Further, due to nonexistent or sporadic monitoring, detailed biological information, including species distributions, is lacking in most Arctic regions. $^{14}$

Hence, it is evident that justifiable OSRA methods for the Arctic need to cope with various uncertainties and limited data. For this reason, the quantitative state-of-the-art methods developed for more extensively studied and data-rich regions are likely not applicable to holistic, large scale OSRA in the Arctic context. Hence, due to rapidly increasing anthropogenic activities and growing societal pressure asking for actions to ensure a sustainable future, we need alternative ways to provide holistic Arctic-applicable risk assessments.

To meet this demand, we propose a novel probabilistic method for conducting OSRA in Arctic marine areas, based on the recently developed probabilistic framework. ${ }^{2}$ The method uses parsimonious probabilistic methods and open access data to hierarchically assess the factors determining the ecological

Received: November 22, 2019

Revised: January 10, 2020

Accepted: January 23, 2020

Published: January 23, 2020 


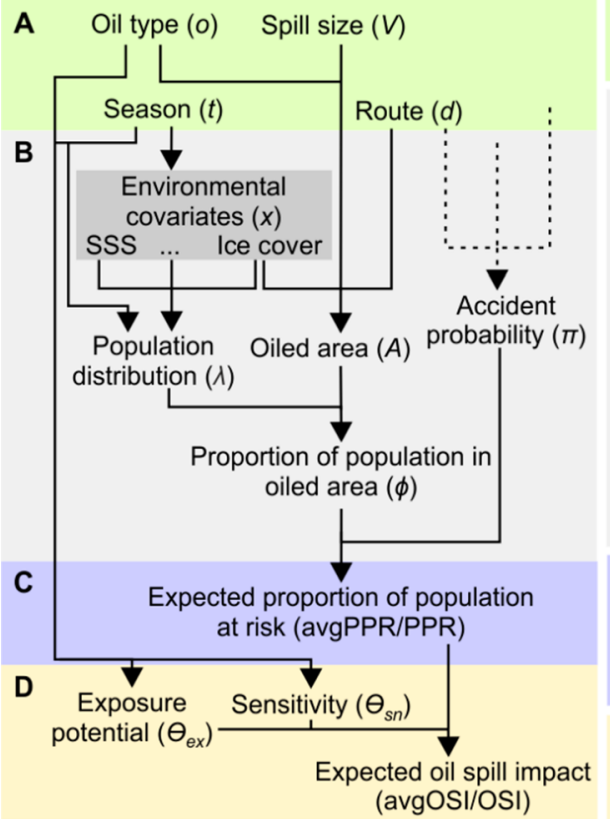

A Oil spill risk can be controlled by choice of shipping season, route, oil type and measures affecting the spill size.
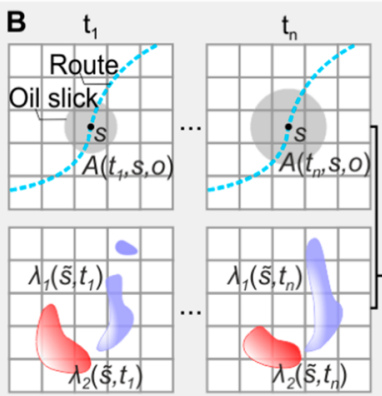

\section{Environmental covariates} vary in space and time affecting species relative density and spreading of oil.

Proportion of population in oiled area after an accident at location $s$

C Expected proportion of population at risk over route, $\rho(d)$, is the expected proportion of population in the oiled area given the probability of an accident, $\pi(\mathrm{s}, \mathrm{t})$, at location $s$ and season $t$ $\operatorname{PPR}(d, t, o)=\int_{s \in P(d)} \phi(s, t, o) \Pi(s, t) d s$

D Biological impact is the product of exposure potantial and sensitivity, $\mathrm{BI}=\Theta_{\mathrm{ex}} \times \theta_{\mathrm{s} n}$. Oil spill impact, OSI $=\mathrm{BI} \times \mathrm{PPR}$, is the expected proportion of population to die after an accident.

Figure 1. Description of the probabilistic oil spill risk assessment method. On the left, a directed acyclic graph (DAG) showing the OSRA factors and their conditional interdependencies. Each factor is a random variable whose probability distribution depends only on the state of the factors preceding it, as indicated by the arrows. The three dashed arrows leading to "Accident probability" illustrate that there are several factors that can affect the probability of an accident (e.g., ice and weather conditions), which were not treated in our case study but could be added into subsequent works. On the right, a description of factors affecting oil spill impact: (A) Factors that can be controlled by management decisions; (B) seasonally and spatially varying factors. Red and purple areas represent population distributions of species 1 and 2, respectively. Environmental covariates and population distribution are stochastic spatiotemporal functions represented as random raster maps that account for environmental stochasticity and parameter uncertainty in species distribution models. The oiled area and the proportion of a population in an oiled area are stochastic spatiotemporal functions represented as random vectors over route points. (C) The expected proportion of a population that is at risk. (D) Spatially constant but seasonally varying species-specific exposure potential and sensitivity and seasonally varying route-specific oil spill impact. Risk measures to be considered: avgPPR $=$ the expected proportion of the population (of a given species) that occurs in the oiled area (i.e., the proportion of the population at risk) when an accident occurs at a random location along a route; PPR $=$ the route-specific risk scaled by the route length; avgOSI = the expected oil spill impact, i.e., the proportion of the population that dies due to oiling when an accident occurs at a random location along a route, defined as avgOSI $=$ avgPPR $\times \mathrm{BI}$; OSI $=$ the route-specific risk scaled by the route length; $\mathrm{BI}=$ biological impact, defined as $\mathrm{BI}=$ exposure potential $\times$ sensitivity.

consequences of oil spills. The pan-Arctic estimates of seasonally varying, species-specific exposure potentials and sensitivities ${ }^{15}$ are estimated separately from seasonally varying species-specific spatial population distributions and the seasonally and spatially varying spreading of oil. This allows risk assessments at various levels of spatial and temporal resolution and the exchange of information between Arctic regions and eases the process of updating assessments with new evidence.

We use our method to assess the risks oil spills pose to the marine environment along the main shipping routes in the Kara Sea (KS). The Northern Sea Route (NSR), which runs through the KS, has received a growing interest as a potentially competitive alternative to shipping through the Suez Canal. ${ }^{16}$ We provide generally applicable quantitative results on the acute impact of oil on adult populations of three arctic marine mammal (AMM) species: polar bear (Ursus maritimus), ringed seal (Pusa hispida), and walrus (Odobenus rosmarus). Further, we estimate the impact of a $42000 \mathrm{~m}^{3}$ oil spill on AMMs for 5 shipping routes in the KS and 4 types of spilled oil (light, medium, heavy, and extra heavy oil) during 3 different seasons (spring, summer, and autumn).

\section{MATERIALS AND METHODS}

2.1. Spatiotemporally Explicit OSRA in Arctic Marine Areas. The probabilistic framework for assessing oil spill risks is presented in Figure 1. We defined an oil spill's acute environmental impact as the proportion of the population of a given species within the study region that dies as a result of the spilled oil within 2 weeks (see ref 17 ) after an accident. ${ }^{2}$ Let us first consider that an oil spill occurs at location $s$ during season $t$ and spreads into area $A(s, t, o, V)$ (oiled area, Figure 1), where $o$ denotes the oil type and $V$, the volume of the spill. Further, $\lambda(\tilde{s}, t) d s$ denotes the proportion of the population of a species within a small area $d \tilde{s}$ around a location $\tilde{s}$ during season $t(\lambda(\tilde{s}, t)$ is the relative intensity of a species to be called the species population distribution). The proportion of the population within the oiled area is then $\phi(s, t, o, V, \lambda)=\int_{\tilde{s} \in A(s, t, o, V)} \lambda(\tilde{s}, t) d \tilde{s}$. However, not all individuals are exposed to oil, even if they were in the oiled area, and not all individuals die when oiled. ${ }^{2,15}$ Hence, we denote by $\theta_{\mathrm{ex}}(t, o)$ and $\theta_{\mathrm{sn}}(t, o)$ the species-specific exposure potential (the proportion of the population within the oiled area that gets into contact with the oil) and sensitivity (the proportion of the oiled individuals that die due to the oil), respectively. The proportion of the population that dies due to an oil spill at location $s$ in season $t$ is then $\phi(t, s, o, V) \theta_{\mathrm{ex}}(t, o) \theta_{\mathrm{sn}}(t, o)$. Further, $d$ denotes the route taken by a ship, $\rho(d)$, the path traveled when route $d$ is chosen, and $\pi(s, t, V)$, the probability that an oil spill of size $V$ occurs at location $s$ at time $t$ (Figure 1). The expected proportion of the population that dies due to an oil spill (herein oil spill impact OSI) along route $d$ at season $t$ is then 


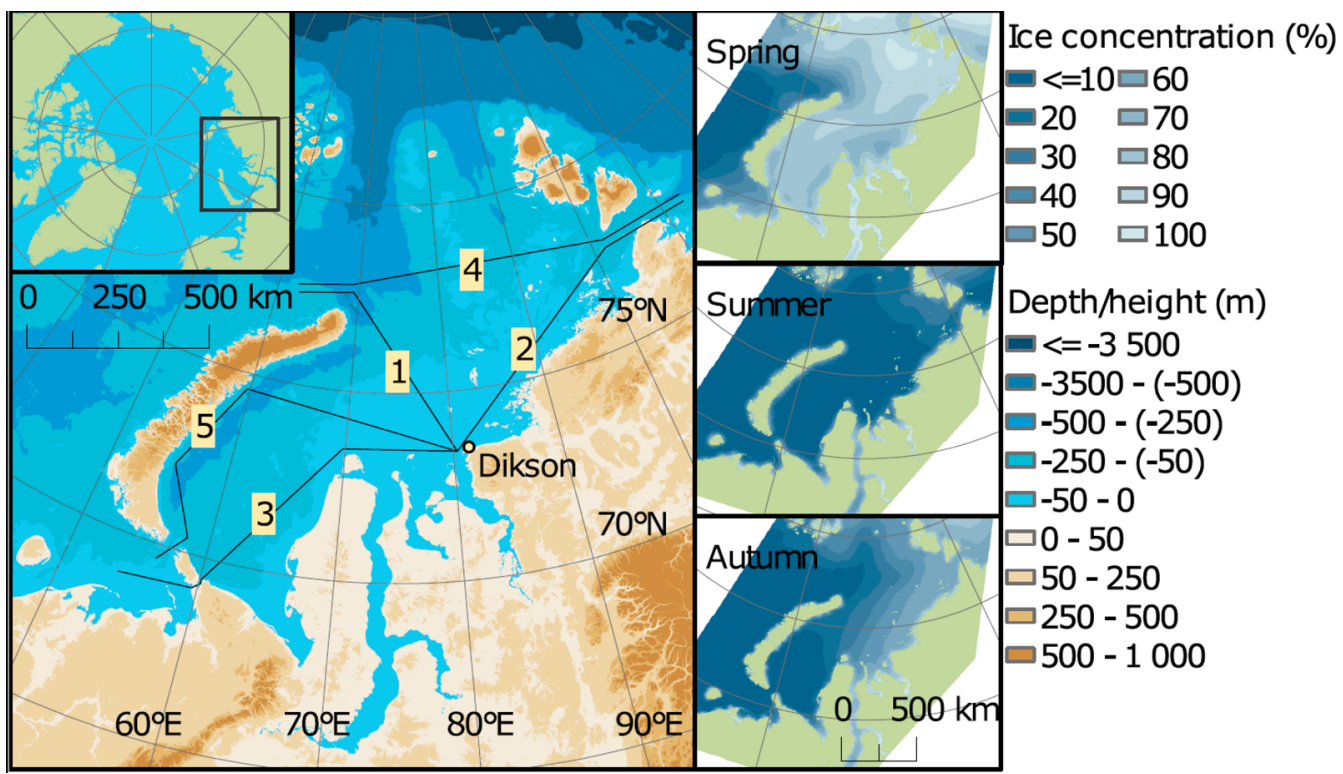

Figure 2. Description of the case study area in the KS. On the left: Bathymetric map of the area and the shipping routes included in the study. On the right: Average ice concentration by season (years 2009-2013).

$$
\begin{aligned}
& \operatorname{OsI}\left(d, t, o, V, \lambda, \theta_{\mathrm{e}}, \theta_{\mathrm{s}}\right)=\theta_{\mathrm{ex}}(t, o) \theta_{\mathrm{sn}}(t, o) \\
& \int_{s \in \rho(d)}\left(\int_{\tilde{s} \in A(s, t, o, V)} \lambda(\tilde{s}, t) d \tilde{s}\right) \pi(s, t, V) d s
\end{aligned}
$$

We call the product $\theta_{\mathrm{ex}}(t, o) \theta_{\mathrm{sn}}(t, o)$ biological impact $(\mathrm{BI})$, and in the general framework, the integral after BI corresponds to the expected proportion of the population at risk (PPR); i.e., PPR is the expected proportion of the population in the oiled area, which may become exposed to oil. The PPR and OSI depend on the length of the route, $|\rho(d)|$, since the probability of an accident along a route increases as the route is lengthened. Hence, as the main risk scores, we examine the routes' average expected proportion of the population at risk and average expected oil spill impact defined as

$$
\operatorname{avgPPR}=\frac{\text { PPR }}{|\rho(d)|}
$$

and

$$
\operatorname{avgOSI}=\frac{\text { OSI }}{|\rho(d)|}
$$

The uncertainties related to the OSRA factors (eq 1, Figure 1) are summarized by probability distributions. When oil type, season, and environmental conditions are known, the processes behind species distribution, accident probability, oil spreading, exposure potential, and sensitivity can be assumed to be mutually independent. This leads to conditional independence between the factors described by the directed acyclic graph (DAG) in Figure 1. Hence, we can factorize, e.g., the joint distribution $p\left(\lambda, A, \pi, \theta_{\mathrm{ex}}, \theta_{\mathrm{sn}} \mid o, t, x, V\right)=p(\lambda \mid t, x) p(A \mid o, x, V) p(\pi \mid$ $o, t, x, V) p\left(\theta_{\mathrm{ex}} \mid o, t\right) p\left(\theta_{\mathrm{sn}} \mid o, t\right)$. The conditional independence structure allows us to first solve each of the conditional probability distributions independently (see Section 2.2) and later combine them. We can solve the (marginal) probability distributions for avgOSI and avgPPR efficiently via Monte Carlo (MC) by sequentially sampling random realizations from the conditional distributions of the OSRA factors (see the Supporting Information). Next, we summarize how we estimated the conditional probability distributions for different
OSRA factors in the case study, which serves also as a demonstration of the practical feasibility of the approach. A more detailed description is included in the Supporting Information.

2.2. Case Study: The Kara Sea. The Kara Sea (KS, Figure 2) is located along the NSR between the Barents and Laptev Seas. It is characterized by a harsh polar maritime climate, with air temperatures below $0{ }^{\circ} \mathrm{C}$ for most of the year. ${ }^{18}$ Ice covers the sea almost completely from October to May and is also present in the summer. The KS has a substantial hydrocarbon resource potential. ${ }^{19}$ As such, the KS may face elevated oil spill risks as a result of increasing traffic along the NSR and increasing oil and gas industry activity in the near future.

We included 5 navigation routes (Figure 2), 3 seasons, and 4 oil types in the analysis and treated the spill size as a scenario variable fixed to an estimate of the Exxon Valdez accident $\left(42000 \mathrm{~m}^{3}\right)$. Seasons were defined according to ref 15 and included spring (March-June), summer (July-September), and autumn (October-November). Winter was omitted, since maritime traffic halts in the KS in winter. ${ }^{20}$ The oil types included light, medium, heavy, and extra heavy oil (see the Supporting Information). The assessment end points were three AMMs: polar bear, ringed seal, and walrus. All three species can be regarded as keystone species in Arctic marine ecosystems, ${ }^{21,22}$ and they are focal ecosystem components (FECs) included in the assessments by the Circumpolar Biodiversity Monitoring Program. ${ }^{14}$

2.2.1. Environmental Covariates. The environmental covariates, $x$, were chosen on the basis of their importance to AMM habitat selection and oil spreading ${ }^{23,24}$ (Table S1). Regarding the latter, the environmental covariate used was sea ice concentration, whereas in species distribution modeling also bathymetry, distance-to-coast, and sea surface salinity (SSS) were applied. Each environmental covariate was represented by monthly raster maps with $5 \mathrm{~km}$ spatial resolution covering the whole KS. Sea ice data were for years 2009-2013, which represents well the current environmental variability in ice conditions $^{25,26}$ (Table S2). The SSS data covered a monthly average over the years 1980-2000 and describe well the 

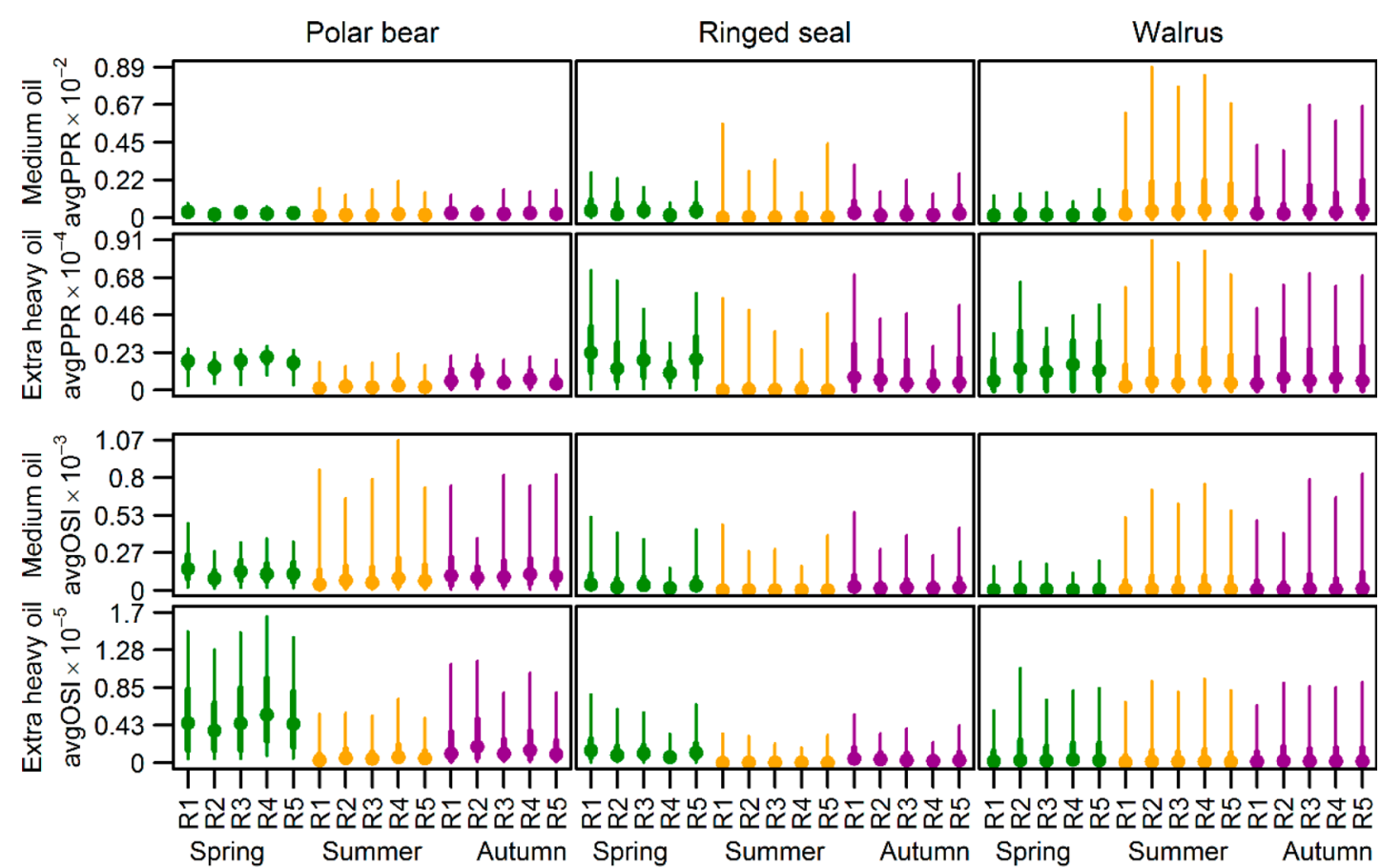

Figure 3. AvgPPRs and avgOSIs for AMM species along R1-R5 with medium (rows 1 and 3) and extra heavy (rows 2 and 4) oil in spring (green), summer (orange), and autumn (purple). Circles denote medians; thick lines are the $25 \%$ to $75 \%$ quantile, and thin lines the $5 \%$ to $95 \%$ quantile.

seasonal variation of SSS and the area of fresh and nutrient rich water in the $\mathrm{KS} .^{25}$ All the data were collected from open source data repositories. ${ }^{25,27,28}$ The uncertainty in environmental covariates in a season was accounted for by an MC sample of environmental covariates corresponding to a randomly drawn month of a specific season. This results in an empirical distribution for $x$.

2.2.2. Population Distribution. We estimated the species' population distribution $\lambda(s, t)$ within the KS by using the probabilistic relative density maps for polar bears, ringed seals, and walrus constructed with the species distribution models described in ref 23. The models describe species areal densities in relation to environmental covariates, location, and time. The biological and environmental data used in the analysis were derived from scientific publications and open source data sets ${ }^{23}$ (Tables S2 and S3). The MC samples for species population distribution were drawn from Bayesian posterior distributions jointly across all grid cells in the KS. For each MC sample, we used the corresponding MC sample of environmental covariates. Hence, the MC samples of $\lambda(s, t)$ reflect the uncertainty originating from both environmental variability and uncertainty about the species' responses to their environment described by model parameter uncertainty.

2.2.3. Oiled Area. The oiled area, $A(s, t, o, V)$, depends on oil type, spill volume, and spatiotemporally varying ice concentration, all of which affect oil spreading. We used Fay-type equations $^{24,29}$ to describe the spreading process as a circular slick (see Figure S5). The parameters of the equations were fixed to values that are representative to the oil types in our analyses (Table S4). However, to account for environmental variability in an oiled area, we formed an MC sample of $A(s, t, o, V)$ for each route point and season using the $\mathrm{MC}$ samples of environmental covariates. The environmental covariates in MC samples of $A(s$, $t, o, V)$ matched the environmental covariates in the corresponding MC samples of $\lambda(s, t)$, so that the (marginal) dependence between $A(s, t, o, V)$ and $\lambda(s, t)$ due to shared environmental conditions is reflected in their joint MC samples.

2.2.4. Accident Probability. As there are no estimates available for accident probabilities in the KS, we fixed the accident probability to a constant across the study area. Hence, avgPPR and avgOSI correspond to the expected proportion of the population at risk and the expected oil spill impact under the assumption that an accident occurs at a random location along a route.

2.2.5. Exposure Potential and Sensitivity. The literature on the exposure potentials and sensitivities of Arctic species is limited. ${ }^{2}$ For ringed seals, we used the previously published probability distributions for $\theta_{\mathrm{ex}}$ and $\theta_{\mathrm{sn}}{ }^{15}$ For polar bears and walrus, we estimated the probability distributions by conducting an extensive literature review (see Table S6) and then turning that information into probability distributions for $\theta_{\mathrm{ex}}$ and $\theta_{\mathrm{sn}}$ with the elicitation tool built by Nevalainen et al. ${ }^{15}$

\section{RESULTS AND DISCUSSION}

We used the following measures to assess oil spill risk for each AMM species across season, oil type, and shipping route (Table S7):

(1) avgPPR: the expected proportion of the population of a given species that occurs in the oiled area and may thus become exposed to oil (i.e., the proportion of the population at risk) when an accident occurs at a random location along a route.

(2) avgOSI: the expected oil spill impact, i.e., the proportion of the population of a given species that dies due to oiling when an accident occurs at a random location along a route, defined as avgOSI $=\operatorname{avgPPR} \times \mathrm{BI}(\mathrm{BI}$ : biological impact $=$ exposure potential $\times$ sensitivity).

(3) avgPPR cmb and avgOSI cmb: the combined avgPPR and the combined avgOSI, respectively, defined as the 


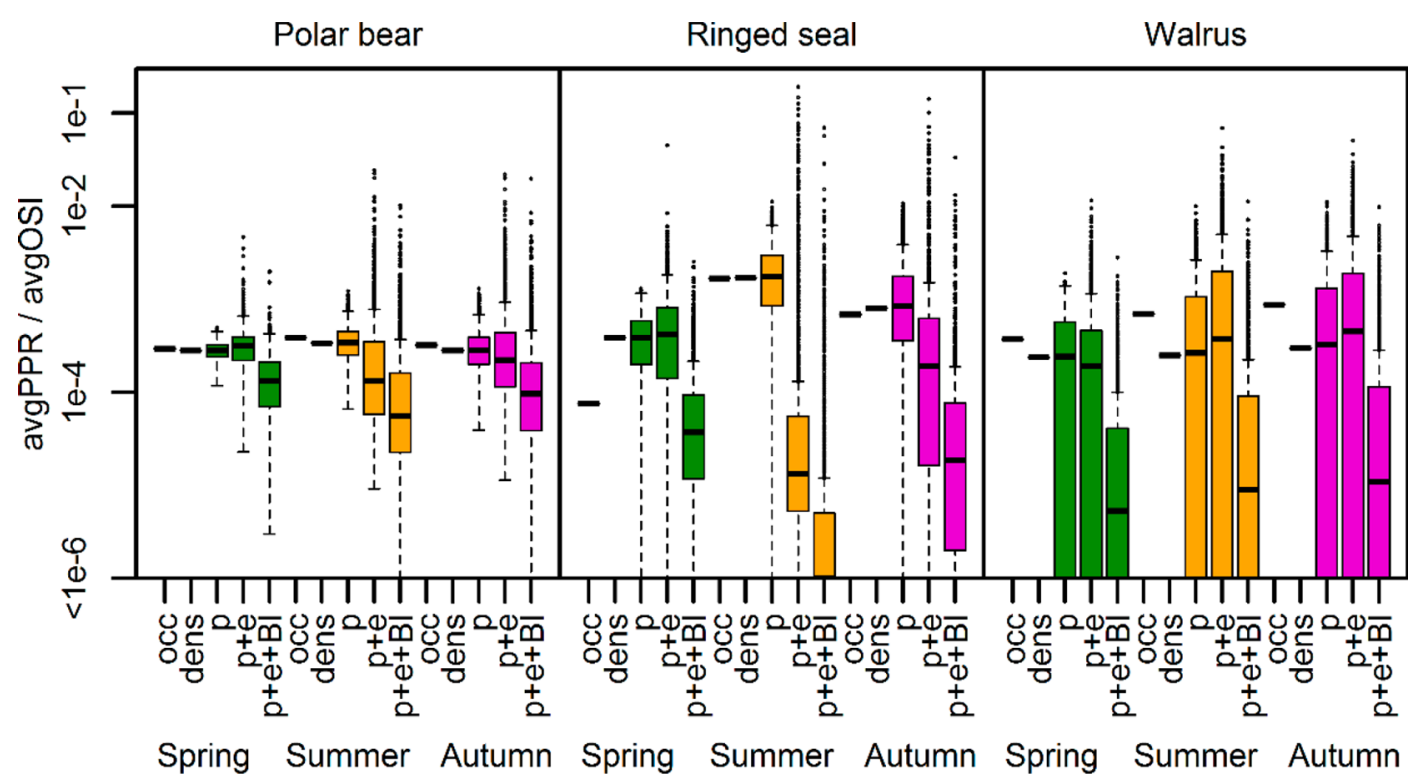

Figure 4. Results with different levels of realism in OSRA factors and sources of uncertainty, when avgPPR (avgOSI in the case of $p+e+\mathrm{BI}$ ) is calculated for medium oil type and route 3. occ: Species occurrence probability is used to describe the population distribution; the population in an oiled area is assumed to die with $100 \%$ certainty $(\mathrm{BI}=1)$; environmental covariates are fixed to their mean values and model parameters to their median values (i.e., we ignore uncertainties in OSRA factors). dens: Species' population distribution is described by its median (i.e., we ignore uncertainty in it); other specifications are the same as in occ. $p$ : Parameter (knowledge) uncertainty is accounted for in population distributions; other specifications are the same as in occ and dens. $p+e$ : Parameter and environmental uncertainty are taken into account in other components except BI, which is assumed to be 1. $p+e+\mathrm{BI}$ : $\mathrm{BI}$ is given the value estimated in the paper; other specifications are the same as in $p+e$. Vertical bars denote the medians; boxes represent the $25 \%$ to $75 \%$ quantile, and whiskers the $5 \%$ to $95 \%$ quantile.

sum of individual avgPPRs and avgOSIs of the three AMM species.

Note that since extra heavy oil spreads inefficiently in all conditions (Figure S5), the avgPPR for extra heavy oil for a given species corresponds to the average proportion of the KS population along the route with the discretization accuracy of 5 $\mathrm{km}$.

3.1. Effect of Season and Oil Type. On average, with respect to season, the distributions of avgPPRs and avgOSIs of polar bears and ringed seals resemble each other. For both species, the distributions of avgPPR and avgOSI are concentrated to their highest values in the spring and the lowest values in the summer. For walrus, avgPPR and avgOSI are the highest in the summer, followed by autumn and spring. With extra heavy oil in autumn, polar bears and walrus have generally higher avgPPRs than ringed seals, whereas in spring, polar bears and ringed seals have higher avgPPRs than walrus; in summer, walrus have the highest avgPPRs (Figures 3 and S9, Table S8). With other oil types, the results are generally the same as above, but the avgPPRs of walrus increase relative to other species in summer and autumn. Further, polar bears have the highest avgOSI across all shipping routes in the KS during every season for all oil types. The avgOSI on ringed seals exceeds the impact on walrus, except during summer (Figures 3 and S11, Table S9).

Further, there are distinct differences between species and seasons in terms of uncertainty in avgPPR and avgOSI. In general, walrus exhibit higher uncertainty than the other two species, and summer and autumn express more uncertainty than spring, which is mostly explained by more variable environmental conditions during summer and autumn than in spring. When uncertainty is high and the probability distributions are hence wide, there is non-negligible probability that the consequences are manifold compared to the most likely (i.e., median) avgPPR and avgOSI. For instance, if light oil is spilled along route 4 in the summer, there is 0.05 probability that the avgPPR of walrus is over 14-fold compared to the median avgPPR (Figure S9, Table S8).

Across all routes and seasons, avgPPRs are the highest for light oil and the lowest for extra heavy oil (Table S8). Lighter oils spread more efficiently than heavier oils both in open water and in ice, exposing greater proportions of AMM populations to oil. However, medium oil has the highest avgOSI across shipping routes and seasons for all species (Table S9), since the species' exposure potential and sensitivity, and hence biological impact (BI), are higher for medium oil compared to light oil (Figures S6-S8).

Although exposure and sensitivity to oil are highly uncertain, they clearly differ between species and oil types (Figures S6S8). Polar bears are sensitive to all oil types but are more likely to become exposed to and exhibit a higher BI with medium and heavy oils than light and extra heavy oils. Ringed seals and walrus are less sensitive to light oil than other oil types. Ringed seals are more exposed to medium and heavy oils than to other oil types, whereas walrus are most exposed to heavy oil. To summarize, the expected impacts to AMM species are lowest for light oil and highest for medium or heavy oil, when only exposure potential and sensitivity to oil are considered.

3.2. Differences between Routes. Typically, routes 1 and 4 exhibit higher avgPPRs and avgOSIs than the other routes. For all three species and all four oil types, in the summer, the highest avgPPR is associated with route 4 . With extra heavy oil, the riskiest routes in spring are routes 1 (ringed seal) and 4 (polar bear and walrus) and in autumn, routes 1 (ringed seal) and 2 (polar bear and walrus). However, with lighter oil, the riskiest routes change partially for polar bear and walrus; e.g., for the former, the highest avgPPR in the spring is associated with route 1 instead of route 4 (Figure 3, Table S8). These changes are due to the different ice conditions in these routes; i.e., although the 
population densities are the highest along route 4 , the oil spreads more efficiently along route 1 than route 4 .

In general, the consideration of avgOSIs instead of avgPPRs does not affect our views on the most risky routes. However, this is not the case when the individual avgPPR and avgOSI scores of the three species are summed up to a combined avgPPR $\mathrm{cmb}$ and avgOSI_cmb (Figures S9 and S11). The avgPPR_cmb does not strongly resemble the avgPPR order for any single species. However, since the polar bears are more sensitive and prone to becoming exposed to oil than the other two species, the order of the routes based on avgOSI_cmb resembles that of avgOSI of polar bears.

We can also assess the routes by using PPR and OSI, which take into account route lengths (e.g., $\mathrm{PPR}=\operatorname{avgPPR} \times|\rho(d)|)$. The highest PPRs and OSIs are associated with routes 5 and 2, which are also the longest routes. With a few exceptions, route 5 has the highest PPR for all species in the spring, whereas in autumn, PPR is the highest for route 2 (Figure S10, Table S10). In the summer, the relative order of routes 5 and 2 differs between species. Again, these changes arise from the differences in ice concentrations along routes and seasons affecting the spreading of oil. The ranking of the route-wise OSIs is the same as that of PPRs (Figure S12, Table S11). The level of uncertainty varies less between routes than between seasons and species. When ranked from the greatest to the smallest PPR or OSI, the order of shipping routes typically differs from the ranking of routes from the longest to the shortest (Table S12). Further, the relative differences in PPRs between routes differ significantly from the relative differences in the route lengths. For instance, although route 1 is only $44 \%$ shorter than route 2 , in the summer, the expected value of PPR of ringed seals is over $96 \%$ lower for route 1 than route 2 . Similarly, although route 5 is the longest, the expected value of its PPR for ringed seals in the summer is only $26 \%$ of the expected value of PPR of route 2 .

3.3. Sources of Uncertainty and the Effect of OSRA Factors. When one accounts for the uncertainty in OSRA factors (Figure 1), there is a clear effect on the analysis, producing different results than when point estimates for model parameters and environmental factors alone are used (Figure 4). All OSRA factors exhibit a high degree of uncertainty. Therefore, as more of these factors are added to the analysis, uncertainty in the avgPPR and avgOSIs accumulates. As stated above, there are clear differences between species and seasons with regard to uncertainty. For all species, a major source of uncertainty is environmental variability (i.e., monthly variation in environmental conditions like sea ice), the inclusion of which widens the probability distributions considerably compared to the cases in which only parameter uncertainty is considered (i.e., uncertainty related to the species' responses to environmental conditions). Uncertainty in biological impact (BI) increases uncertainty in avgPPR and avgOSI even more.

Moreover, the assessment based on species population distribution (areal abundance estimates) differs significantly from a more traditional risk assessment, which is based on the probability of species occurrence alone. Risk estimates based on population distribution can be either higher or lower than estimates based on species occurrence alone (Figure 4). For example, the avgPPR of polar bears calculated with the occurrence probability alone does not differ much from the avgPPR calculated using population distribution; however, for the other two species, the difference is distinct. The reason is that the ringed seal population distribution varies more than that of walrus inside their corresponding high occurrence probability areas (Figures S2 and S3). Therefore, the occurrence probability of ringed seals does not reflect well their population distribution and leads to a too low avgPPR in the spring. In general, population density contains more information than occurrence probability.

It is important to explicitly take uncertainty into account. In environmental risk assessment, we are often interested in extreme or "worst" cases, i.e., the tails of the probability distributions. For instance, we can base our analysis on the $95 \%$ quantiles of the distributions and ask how big impacts will occur with a probability of 0.05 . The adoption of this approach does not necessarily change our understanding; for instance, usually, for the riskiest routes, the order from the least to the most risky route remains the same independent of whether we apply medians or $95 \%$ quantiles (Figures S9-S12). However, there are also situations where these two approaches produce different results and, more importantly, the quantiles have a direct impact on decision-making, since they can be used to define acceptable risk levels.

3.4. Spatiotemporally Varying Oil Spill Risks. Our case study shows that ranking shipping routes according to the risk oil spills pose to the environment is not a straightforward task. We cannot assume that shorter routes equate to lower risks. We can assume neither that heavy oils are always more risky than light oils nor that routes near high population density areas are automatically more risky than routes near low population density areas. Seasonal variation in risk and differences between species and oil types are substantial. It is essential to be aware of these discrepancies, since they indicate that it might be impossible to find an ideal risk management solution for all ecological components present in a given area.

Although the population density of a given species may be high along one route, another route may be riskier if oil spreads more efficiently there, exposing a larger proportion of the population to oil. The lighter the spilled oil, the higher is its spreading capacity and exposure potential (Figure S5). This is especially true in the Arctic, where weathering processes, like evaporation, are typically attenuated (see the Supporting Information). ${ }^{13}$ Heavier oils, on the other hand, pose a higher risk in terms of smothering, although they are usually less toxic and spread less than light oils. ${ }^{30}$ These properties affect the species exposure and sensitivity to oil, which in turn, lead to differences between avgPPR and avgOSI.

These differences would be even more distinct if more species were added to the analysis. For example, seabirds are more exposed and sensitive to medium and heavy oils than to light oils. ${ }^{15}$ Light oils, which typically contain more toxic and soluble components than heavier oils, ${ }^{30}$ would likely pose a greater risk to fish. The inclusion of benthic fauna in the analysis could have emphasized the risk related to extra heavy oils, which may sink. The extension of the focus to long-term impacts would also have stressed the importance of heavy oils, since heavier oils are more persistent in the environment than lighter oils. ${ }^{31}$ Further, our study focuses only on the adult AMMs. For juveniles, the differences between seasons are expected to be more distinct as, e.g., seal pups are especially susceptible to negative effects of oiling until they have accumulated a thick blubber layer to rely on for buoyancy and thermoregulation. ${ }^{32}$

Our results demonstrate the need for more accurate information on species ranges and abundances. For example, both fish and benthos are still poorly monitored in many parts of the Arctic. ${ }^{14}$ Moreover, as demonstrated with Figure 4, additional information on species ranges only would not 
necessarily change our understanding of the safest or riskiest routes and could even lead to misleading risk estimates. If the species' estimated ranges cover large areas overlapping with several shipping routes and we lack information on species abundances, we are not able to differentiate between routes. Further, we are likely to over- or underestimate the route specific risks in a manner that is not systematic over species or seasons. Hence, our results highlight the more general observation that species range estimates alone are inferior to population distribution estimates in management applications. ${ }^{3 \frac{13}{3}}$

3.5. General Lessons for OSRA in the Arctic. Despite our analysis focuses on the KS, it provides three lessons for oil spill risk management generalizable to the Arctic as a whole. First, the results support current aspirations to ban heavy fuel oil (HFO) in the Arctic. ${ }^{34}$ Second, in some situations, light oils, however, pose a higher risk to the environment, as they have higher spreading capacity than heavy oils. Therefore, we should not base our risk assessments solely on the impacts of oiling on individual species (i.e., sensitivity) but also on the exposure capacity of different oil types. This includes both the behavior of the spilled oil itself (i.e., to what extent oil contaminates different parts of the ecosystem) and the probability of organisms to become exposed to oil. Although the understanding of the fate of oil in ice has improved in recent years, ${ }^{11,12,35}$ there is still a need for a deeper understanding of the weathering processes that oil undergoes. These processes contribute to the exposure capacity and persistence of oil in the environment, which influence the short-term and long-term effects of oil spills. ${ }^{2,30}$ Third, our results highlight the importance of season-specific OSRA in the Arctic. Many of the physical factors influencing Arctic biota, such as sea ice, vary seasonally and interannually. ${ }^{36}$ Population distributions change in accordance with these variations, resulting in varying risk levels. For example, in the KS, the shipping routes receiving the highest and lowest avgPPRs for ringed seals differ between seasons, and the situation in the summer can be the opposite of the situation in spring and autumn (Table S8). Therefore, we cannot base OSRA on areal species densities averaged over seasons, as they might lead to erroneous conclusions about risk level.

3.6. Benefits and Drawbacks of the Developed Method. The approach presented here has several advantages. First, it provides quantitative estimates of spatiotemporally varying oil spill risk, while expressing uncertainty explicitly. This is important since proper quantification of uncertainty should be of paramount interest when the aim of risk assessment is to support decision-making. ${ }^{37}$ This is especially true in the Arctic, where underlying uncertainties are typically large. ${ }^{38}$ Our study confirms this point and demonstrates that uncertainty can differ considerably between seasons, oil types, and species.

Second, the method offers a relatively simple way to make justifiable risk comparisons over large geographic areas. Prior OSRA methods typically use trajectory models to describe the spread and transport of spilled oil. Then, they combine this information with knowledge about the resources at risk. The realism and spatial resolution of trajectory models are higher than those of our oil-spreading model. However, it is not usually feasible to conduct trajectory modeling with many potential spill locations, and therefore, the assessment is usually done for only a few spill locations at most (see, e.g., refs 5 and 39-41). Naturally, such an approach is suboptimal when comparing shipping routes that are hundreds of kilometers long. By considering each grid cell $(5 \times 5 \mathrm{~km})$ along a route as the potential origin of an oil spill, our method assesses risk more comprehensively than is possible when only a few spill locations are considered. This level of resolution also pairs well with the broad-scale environmental data available for the Arctic. We believe that methods that allow OSRA over large areas can also support marine spatial planning, which will become increasingly important when new opportunities for shipping, oil and gas exploitation, fisheries, and tourism open in the Arctic. ${ }^{42}$

Third, by taking spatiotemporal variation in population distributions into account, we can provide information on a meaningful scale for decision-making. The purpose of any environmental risk assessment (ERA) is to provide support for decision-making. ${ }^{43}$ In order to achieve this goal, ERAs should offer decision makers information that enables them to make comparisons between different management alternatives. Thus far, the risks that maritime activities pose to Arctic marine species have mainly been determined at a relatively coarse level, by either qualitative (e.g., ref 1 ) or semiquantitative means. ${ }^{44}$ Although such approaches provide a good overall view of the spatial distributions of the ecological components at risk, their utility in the decision-making context is limited, since their coarse resolution does not enable the differentiation between areas (see, e.g., ref 44).

Fourth, our approach handles variations in risk related to oil types and seasons more comprehensively than previous assessment methods. Typically, oil spill modeling studies take oil type and season into account in terms of their effects on oil behavior by simulating the fate and trajectories of spilled oil under season-specific weather conditions, but their effects on ecological components are often limited to, e.g., seasonal (e.g., refs 9 and 44) or daily ${ }^{5}$ changes in the species' ranges (but see ref 45 ). However, in addition to spatiotemporally varying species' ranges, our results emphasize the importance of accounting for season and oil type in season- and oil type-specific exposure potentials and sensitivities.

The greatest limitations of our analysis are that it excludes the transport of oil by currents, ice, etc. and does not consider oil weathering. The consequences of these simplifications are season-specific and depend largely on ice cover and, to some extent, oil type. The impact of ice on the movement of oil is considered negligible until the ice covers $30 \%$ of a given area. When ice cover exceeds $80 \%$, the oil is assumed to be contained within the ice and hence be transported with it. ${ }^{46}$ By leaving oil transport out of our analysis, we likely underestimate the oiled area, especially under low-ice conditions, which explains our low avgPPRs. This underestimation is counteracted, at least to some extent, by the omission of evaporation, which typically decreases the amount of oil on the sea surface, although evaporation is reduced in icy conditions and at low temperatures (see the Supporting Information and ref 11). To summarize, albeit the oiled area may be somewhat underestimated, our analysis provides realistic estimates of risk and associated uncertainties in it. This possible underestimation does not directly affect our conclusions about the riskiest shipping routes if we assume that, on average, the transport of oil does not differ extensively between routes. We believe that this assumption is valid on the scale of our study but might not apply if we compare routes that are located very far from each other and that exhibit very different ice conditions. Hence, there is an apparent need for information on currents and ice movements as well as for computationally cheap methods to simulate oil spreading under those conditions for a large number of spill locations.

Moreover, as the analysis focuses on the acute impacts of oil spills, some important ecological aspects are not taken into 
account. For example, population decline is believed to be the most detrimental for species that mature late and have few offspring. ${ }^{47}$ Hence, polar bears and walrus, which do not breed annually, may suffer more over time than ringed seals, which do. Furthermore, walrus may be particularly susceptible to the chronic impacts of oiling, since their preferred prey, mussels, are known to accumulate oil-derived toxins. Oil spills may also have long-term indirect effects by inducing changes in food webs. ${ }^{47}$ However, both the long-term fate of spilled oil and its ecological effects are virtually impossible to predict using the current methods. ${ }^{2}$

Our study does not acknowledge differences in the ecological or economic value between species but, rather, weights species equally. There are different ways to value biota (see, e.g., ref 48), for example, on the basis of conservation status. As an example, polar bear and walrus are regarded as "vulnerable" and ringed seals, as "least concern" according to the IUCN Red List. ${ }^{49}$ This information could be taken into account in OSRA by weighting the value of each species according to their IUCN status. ${ }^{39}$

Lastly, the route-specific results are conditioned on the constant accident probability and fixed spill size. Hence, the results encompass the consequence analysis component of OSRA but must be supplemented with oil spill probabilities to be considered comprehensive. The extension of the analysis to cover spatiotemporally varying accident probabilities is relatively straightforward if such assessments become available. This would probably underline even more the fact that we should not base our conclusions solely on the route lengths, as a ship sailing on a shorter route exhibiting harsh weather conditions may face higher accident probability than a ship sailing a longer route in less challenging conditions. Hence, the supplementation of the analysis with spill probabilities is strongly supported. This holds true also for uncertainty related to spill size, for which probabilistic methods have been developed (see, e.g., ref 50).

\section{ASSOCIATED CONTENT}

\section{SI Supporting Information}

The Supporting Information is available free of charge at https://pubs.acs.org/doi/10.1021/acs.est.9b07086.

Additional materials and methods; expected relative densities of polar bears, walrus, and ringed seals; example of the overlap of the oil slick and the species densities at high and low ice concentrations; environmental covariates and the justification for their inclusion in the model; specifications of the environmental covariates used in the study; species data used in the study; oil spreading under different ice cover conditions; parameter values used in oil spreading calculations; oil slick areas and ratios between different oil types with and without evaporation; reasoning and references related to the exposure potential and sensitivity of the species; probability distributions for polar bear, ringed seal, and walrus exposure potential, sensitivity, and the biological impact of oil; risk measures used in the analysis; avgPPR, PPR, avgOSI, and OSI analyses for AMM species (PDF)

\section{AUTHOR INFORMATION}

\section{Corresponding Authors}

Inari Helle - Ecosystems and Environment Research Programme, Faculty of Biological and Environmental Sciences and Helsinki Institute of Sustainability Science (HELSUS), University of
Helsinki, University of Helsinki FI-00014, Finland; ○ orcid.org/0000-0001-9006-0899; Email: inari.helle@ helsinki.fi

Jarno Vanhatalo - Organismal and Evolutionary Biology Research Programme, Faculty of Biological and Environmental Sciences and Department of Mathematics and Statistics, Faculty of Science, University of Helsinki, University of Helsinki FI00014, Finland; O orcid.org/0000-0002-6831-0211; Email: jarno.vanhatalo@helsinki.fi

\section{Authors}

Jussi Mäkinen - Organismal and Evolutionary Biology Research Programme, Faculty of Biological and Environmental Sciences, University of Helsinki, University of Helsinki FI-00014, Finland; (1) orcid.org/0000-0001-6599-8279

Maisa Nevalainen - Organismal and Evolutionary Biology Research Programme, Faculty of Biological and Environmental Sciences, University of Helsinki, University of Helsinki FI-00014, Finland

Mawuli Afenyo - Transport Institute, University of Manitoba, Winnipeg, Manitoba R3T 5V4, Canada; ○ orcid.org/00000002-3094-5617

Complete contact information is available at:

https://pubs.acs.org/10.1021/acs.est.9b07086

\section{Notes}

The authors declare no competing financial interest.

\section{ACKNOWLEDGMENTS}

This work was funded by the Lloyd's Register Foundation (Agreement 28/5/2013). The LRF supports the advancement of engineering-related education and funds research and development that enhances the safety of life at sea, on land, and in the air. I.H. was also funded by the Helsinki Institute of Sustainability Science (HELSUS); M.A. was funded by the GENICE project (Microbial Genomics for Oil Spill Preparedness in Canada's Arctic Marine Environment), and J.M. and J.V. were funded by the Academy of Finland (grants 304531 and 317255) and the University of Helsinki research funds. The authors wish to thank Kelsey La Mere, Floris Goerlandt, Sakari Kuikka, Samu Mäntyniemi, and Laura Uusitalo for their helpful comments on the manuscript.

\section{REFERENCES}

(1) AMAP. Assessment 2007: Oil and Gas Activities in the Arctic - Effects and Potential Effects; Arctic Monitoring and Assessment Programme: Oslo, Norway, 2010; Vol. 1.

(2) Nevalainen, M.; Helle, I.; Vanhatalo, J. Preparing for the unprecedented - Towards quantitative oil risk assessment in the Arctic marine areas. Mar. Pollut. Bull. 2017, 114 (1), 90-101.

(3) Arzaghi, E.; Abbassi, R.; Garaniya, V.; Binns, J.; Khan, F. An ecological risk assessment model for Arctic oil spills from a subsea pipeline. Mar. Pollut. Bull. 2018, 135, 1117-1127.

(4) Carroll, J.; Vikebo, F.; Howell, D.; Broch, O. J.; Nepstad, R.; Augustine, S.; Skeie, G. M.; Bast, R.; Juselius, J. Assessing impacts of simulated oil spills on the Northeast Arctic cod fishery. Mar. Pollut. Bull. 2018, 126, 63-73.

(5) Wilson, R. R.; Perham, C.; French-McCay, D. P.; Balouskus, R. Potential impacts of offshore oil spills on polar bears in the Chukchi Sea. Environ. Pollut. 2018, 235, 652-659.

(6) Wenning, R. J.; Robinson, H.; Bock, M.; Rempel-Hester, M. A.; Gardiner, W. Current practices and knowledge supporting oil spill risk assessment in the Arctic. Mar. Environ. Res. 2018, 141, 289-304. 
(7) Frazão Santos, C.; Michel, J.; Neves, M.; Janeiro, J.; Andrade, F.; Orbach, M. Marine spatial planning and oil spill risk analysis: Finding common grounds. Mar. Pollut. Bull. 2013, 74 (1), 73-81.

(8) Spaulding, M. L. State of the art review and future directions in oil spill modeling. Mar. Pollut. Bull. 2017, 115 (1), 7-19.

(9) French McCay, D. P. Oil spill impact modeling: Development and validation. Environ. Toxicol. Chem. 2004, 23 (10), 2441-2456.

(10) Reed, M.; Aamo, O. M.; Daling, P. S. Quantitative Analysis of Alternate Oil-Spill Response Strategies Using Oscar. Spill Sci. Technol. Bull. 1995, 2 (1), 67-74.

(11) Afenyo, M.; Veitch, B.; Khan, F. A state-of-the-art review of fate and transport of oil spills in open and ice-covered water. Ocean Eng. 2016, 119, 233-248.

(12) French-McCay, D. P.; Tajalli-Bakhsh, T.; Jayko, K.; Spaulding, M. L.; Li, Z. K. Validation of oil spill transport and fate modeling in Arctic ice. Arctic Science 2017, 4 (1), 71-97.

(13) Nordam, T.; Beegle-Krause, C. J.; Skancke, J.; Nepstad, R.; Reed, M. Improving oil spill trajectory modelling in the Arctic. Mar. Pollut. Bull. 2019, 140, 65-74.

(14) CAFF. State of the Arctic Marine Biodiversity Report; Conservation of Arctic Flora and Fauna International Secretariat: Akureyri, Iceland, 2017.

(15) Nevalainen, M.; Helle, I.; Vanhatalo, J. Estimating the acute impacts of Arctic marine oil spills using expert elicitation. Mar. Pollut. Bull. 2018, 131, 782-792.

(16) Liu, M.; Kronbak, J. The potential economic viability of using the Northern Sea Route (NSR) as an alternative route between Asia and Europe. J. Transp. Geogr. 2010, 18 (3), 434-444.

(17) Boehm, P. D.; Page, D. S. Exposure elements in oil spill risk and natural resource damage assessments: A review. Hum. Ecol. Risk Assess. 2007, 13 (2), 418-448.

(18) Marchenko, N. Russian Arctic Seas: Navigational conditions and accidents; Springer: New York, U.S., 2012.

(19) Gautier, D. L.; Bird, K. J.; Charpentier, R. R.; Grantz, A.; Houseknecht, D. W.; Klett, T. R.; Moore, T. E.; Pitman, J. K.; Schenk, C. J.; Schuenemeyer, J. H.; Sørensen, K.; Tennyson, M. E.; Valin, Z. C.; Wandrey, C. J. Assessment of Undiscovered Oil and Gas in the Arctic. Science 2009, 324 (5931), 1175-1179.

(20) Khon, V. C.; Mokhov, I. I.; Latif, M.; Semenov, V. A.; Park, W. Perspectives of Northern Sea Route and Northwest Passage in the twenty-first century. Clim. Change 2010, 100 (3-4), 757-768.

(21) Smith, T. G.; Hammill, M. O.; Taugbol, G. A Review of the Developmental, Behavioral and Physiological Adaptations of the Ringed Seal, Phoca Hispida, to Life in the Arctic Winter. Arctic 1991, 44 (2), 124-131.

(22) Tryland, M.; Lydersen, C.; Kovacs, K. M.; Thoresen, S. I. Serum chemistry reference values in free-ranging North Atlantic male walruses (Odobenus rosmarus rosmarus) from the Svalbard archipelago. Vet. Clin. Pathol. 2009, 38 (4), 501-506.

(23) Mäkinen, J.; Vanhatalo, J. Hierarchical Bayesian model reveals the distributional shifts of Arctic marine mammals. Divers. Distrib. 2018, 24 (10), 1381-1394.

(24) S.L. Ross Environmental Research Limited; D.F. Dickins Associates Limited Field Research Spills to Investigate the Physical and Chemical Fate of Oil in Pack Ice; Environmental Studies Revolving Funds Report 062; Environmental Studies Research Funds: Ottawa, Canada, 1987.

(25) Mäkinen, J.; Vanhatalo, J. Hydrographic responses to regional covariates across the Kara Sea. J. Geophys. Res.- Oceans 2016, 121 (12), $8872-8887$.

(26) Divine, D. V.; Korsnes, R.; Makshtas, A. P.; Godtliebsen, F.; Svendsen, H. Atmospheric-driven state transfer of shore-fast ice in the northeastern Kara Sea. J. Geophys. Res. 2005, 110 (C9), C09013.

(27) Cavalieri, D.; Parkinson, C.; Gloersen, P.; Zwally, H. J.; National Snow and Ice Data Center Sea ice concentrations from Nimbus-7 SMMR and DMSP SSM/I passive microwave data; NASA National Snow and Ice Data Center Distributed Active Archive Center: Boulder, Colorado, USA, 1996; DOI: 10.5067/8GQ8LZQVLOVL (deposited 29 September 2015).
(28) Jakobsson, M.; Mayer, L.; Coakley, B.; Dowdeswell, J. A.; Forbes, S.; Fridman, B.; Hodnesdal, H.; Noormets, R.; Pedersen, R.; Rebesco, M.; Schenke, H. W.; Zarayskaya, Y.; Accettella, D.; Armstrong, A.; Anderson, R. M.; Bienhoff, P.; Camerlenghi, A.; Church, I.; Edwards, M.; Gardner, J. V.; Hall, J. K.; Hell, B.; Hestvik, O.; Kristoffersen, Y.; Marcussen, C.; Mohammad, R.; Mosher, D.; Nghiem, S. V.; Pedrosa, M. T.; Travaglini, P. G.; Weatherall, P. The International Bathymetric Chart of the Arctic Ocean (IBCAO) Version 3.0. Geophys. Res. Lett. 2012, 39, L12609.

(29) Fay, J. A. The Spread of Oil Slicks on a Calm Sea. In Oil on the Sea; Hoult, D. P., Ed.; Plenum Press: New York, 1969; pp 53-63.

(30) NRC. Oil in the Sea III: Inputs, Fates, and Effects; National Academic Press: Washington, DC, 2003.

(31) Mackay, D.; McAuliffe, C. D. Fate of hydrocarbons discharged at sea. Oil Chem. Pollut. 1989, 5 (1), 1-20.

(32) St. Aubin, D. J. Physiologic and Toxic Effects on Pinnipeds. In Sea Mammals and Oil: Confronting the Risks; Geraci, J. R., St. Aubin, D. J., Eds.; Academic Press: San Diego, 1990; pp 103-127.

(33) Kallasvuo, M.; Vanhatalo, J.; Veneranta, L. Modeling the spatial distribution of larval fish abundance provides essential information for management. Can. J. Fish. Aquat. Sci. 2017, 74 (5), 636-649.

(34) Prior, S.; Walsh, D. A Vision for a Heavy Fuel Oil-Free Arctic. Environment 2018, 60 (6), 4-11.

(35) Fingas, M. F.; Hollebone, B. P. Oil behaviour in ice-infested waters. In Proceedings of the 36th AMOP Technical Seminar on Environmental Contamination and Response, 2013; pp 110-135.

(36) Kovacs, K. M.; Lydersen, C.; Overland, J. E.; Moore, S. E. Impacts of changing sea-ice conditions on Arctic marine mammals. Mar. Biodivers. 2011, 41 (1), 181-194.

(37) Burgman, M. A.; Yemshanov, D. Risks; decisions and biological conservation. Divers. Distrib. 2013, 19 (5-6), 485-489.

(38) Emmerson, C.; Lahn, G. Arctic Opening: Opportunity and Risk in the High North Project Report; Lloyd's and Chatham House: London, 2012.

(39) Helle, I.; Jolma, A.; Venesjärvi, R. Species and habitats in danger: estimating the relative risk posed by oil spills in the northern Baltic Sea. Ecosphere 2016, 7 (5), No. e01344.

(40) Romero, A. F.; Abessa, D. M. S.; Fontes, R. F. C.; Silva, G. H. Integrated assessment for establishing an oil environmental vulnerability map: Case study for the Santos Basin region; Brazil. Mar. Pollut. Bull. 2013, 74 (1), 156-164.

(41) Nordam, T.; Dunnebier, D. A. E.; Beegle-Krause, C. J.; Reed, M.; Slagstad, D. Impact of climate change and seasonal trends on the fate of Arctic oil spills. Ambio 2017, 46 (Suppl3), 442-452.

(42) Edwards, R.; Evans, A. The challenges of marine spatial planning in the Arctic: Results from the ACCESS programme. Ambio 2017, 46 (Suppl 3), 486-496.

(43) Burgman, M. Risks and Decisions for Conservation and Environmental Management; Cambridge University Press: Cambridge, 2005.

(44) Hauser, D. D. W.; Laidre, K. L.; Stern, H. L. Vulnerability of Arctic marine mammals to vessel traffic in the increasingly ice-free Northwest Passage and Northern Sea Route. Proc. Natl. Acad. Sci. U. S. A. 2018, 115 (29), 7617-7622.

(45) Lecklin, T.; Ryömä, R.; Kuikka, S. A Bayesian network for analyzing biological acute and long-term impacts of an oil spill in the Gulf of Finland. Mar. Pollut. Bull. 2011, 62 (12), 2822-2835.

(46) Afenyo, M.; Khan, F.; Veitch, B.; Yang, M. Modeling oil weathering and transport in sea ice. Mar. Pollut. Bull. 2016, 107 (1), 206-215.

(47) Chapman, P. M.; Riddle, M. J. Toxic effects of contaminants in polar marine environments. Environ. Sci. Technol. 2005, 39 (9), 200A206A.

(48) Laurila-Pant, M.; Lehikoinen, A.; Uusitalo, L.; Venesjärvi, R. How to value biodiversity in environmental management? Ecol. Indic. 2015, 55, 1-11.

(49) IUCN. The IUCN Red List of Threatened Species; Version 2018-2. http://www.iucnredlist.org (acceded 15 January 2019). 
(50) Goerlandt, F.; Montewka, J. A probabilistic model for accidental cargo oil outflow from product tankers in a ship-ship collision. Mar. Pollut. Bull. 2014, 79 (1-2), 130-44. 